e-Neuroforum $2011 \cdot 2: 6-12$

DOI 10.1007/s13295-011-0014-5

(C) Springer-Verlag 2011

\author{
J.S. Wiegert $\cdot$ T.G. Oertner \\ Friedrich Miescher Institute for Biomedical Research, Basel
}

\title{
Shapeshifting for memory
}

\author{
Biochemical and electrical \\ signaling in dendritic spines
}

\section{Introduction}

Since the discovery of dendritic spines more than 100 years ago by Ramón y Cajal, there has been intense debate about their function. Today, thanks to advanced microscopy methods, we are able to record functional signals from single spines in intact brain tissue. Here we review recent discoveries that point to a critical function of spines in the regulation of synaptic strength. We would even argue that spines and the synapses impinging on them are the elementary units of memory. But let us start with a definition: what exactly are spines?

\section{Spines are morphologically diverse; their micro- anatomy is correlated with synaptic properties}

Spines protrude directly from the dendritic shaft of neurons; they consist of a bulbous head that is connected to the dendrite by a thin stalk, the spine neck. The fine structure of spines is highly variable, even on the dendrite of a single neuron, making it difficult to classify them. The most common classification is into thin, stubby, and mushroom-shaped spines. Mushroom spines have a large head, thin neck, and are frequently found in the adult brain, whereas stubby spines have no discernable neck. In addition, thin filopodia exist on dendrites that lack some of the properties of true spines and are generally assumed to be immature predecessors. It should be noted, however, that this classification has been invented to order a continuum of shapes, making defini- tions somewhat arbitrary. In addition, as we will explain in detail later, spine structure is not always stable, but morphology (and function) can change rapidly depending on neuronal activity. Therefore, the distribution of spines on the dendrites of a neuron constantly changes and represents merely a snapshot of cellular connectivity and synaptic properties at a certain point in time.

In spite of their morphological diversity, spines have a number of common properties: First, they are very small. Their length is less than $3 \mu \mathrm{m}$, with a maximum diameter of about $1.5 \mu \mathrm{m}$. The volume of the spine head is typically 0.05 femtoliter (0.05 $\times 10^{-15}$ liter). Since this tiny volume is diffusionally isolated from the much larger dendrite by the spine neck, it provides a restricted space for biochemical reactions. The extremely small volume suggests that neurons have miniaturized their synaptic receivers to cram as many connections as possible into a limited space. In addition, the small volume might have advantages for efficient processing of synaptic signals. It is a useful analogy to view the spine as a nanoreactor, able to sustain restricted biochemical reactions with minimal amounts of substrate. Next, we would like to explain how processing of synaptic signals might profit from the small volume and the narrow neck of dendritic spines.

Typically, every spine forms a single excitatory synaptic contact with a presynaptic axon. The few 'orphan' spines without a synaptic contact are small and lack a true head. They probably represent a small population of spines that are either newly formed or in the process of retraction after losing contact to the presynaptic bouton. Spines with a presynaptic partner contain a specialized protein network in their heads, termed the 'postsynaptic density' (PSD) due to its dark appearance in the electron microscope. Most proteins in the PSD are directly or indirectly involved in communicating between neurons and in the regulation of synaptic strength. The size of the PSD is strongly correlated with the total number of presynaptic vesicles and with the number of vesicles released during presynaptic activity. Typically, a synapse formed on a large spine has a large PSD and releases more neurotransmitter than a connection impinging on a small spine. In addition, large spines with large PSDs contain many glutamate receptors of the AMPA-type and are thus in a position to depolarize the dendrite more strongly [1]. These correlations suggest that we can estimate the strength of a synapse, and thus its impact on the neuronal network, from the structure of a spine.

In rare cases, spines with multiple heads have been reported. These forked spines are in contact with two presynaptic boutons, usually formed by two different axons. This rule also applies to neighboring spines on a dendritic segment, which are almost always contacted by axons from different cells. This distributed input allows the neuron to receive and integrate information from a very large number of presynaptic neurons (fan-in). The distribution of spine morphologies along a dendrite is very heterogeneous: it is not possible to deduce from the shape of a spine its position in the dendritic tree. Distances between spines are also quite variable. These observations are consistent with regulation of morphology on the level of 
individual spines. There is, however, a tendency for spines on distal parts of the dendritic tree to be larger, at least on CA1 pyramidal cells. The greater strength of distal synapses, far away from the cell body, might be necessary to compensate for the attenuation of synaptic signals during dendritic propagation.

\section{Dynamic structural changes and their functional significance}

Since the advent of modern laser scanning microscopy (• Fig. 1), spines can be resolved with good spatial and temporal resolution in intact tissue. Time-lapse observations have shown that spines are in constant movement. This motility is especially important in the young brain, when synaptic connections are being formed and optimized. Thin spines and their predecessors, the filopodia, search for active axons in their environment. This exploratory activity illustrates a key function of spines: they vastly increase the space in which synaptic contacts with presynaptic axons can be established. Therefore, a presynaptic axon is not required to directly touch the dendrite of the postsynaptic cell to form a synapse. This interpretation is corroborated by the observation that axons are relatively immobile and straight: they do not meander from synapse to synapse. This has the advantage that the presynaptic cell is able to achieve the same connectivity using less 'cable', thus saving material and accelerating signal propagation. Hence, the high motility of young spines enables the postsynaptic cell to form as many contacts as possible and, likewise, to sample the putative contacts functionally in order to stabilize relevant connections only.

As stated above, the appearance and disappearance of filopodia and spines is especially pronounced in early development, when the synaptic connections of individual brain regions are being refined. However, spine motility is also important in the adult brain [2]. Spines can change their morphology within seconds [3] and the volume of spine heads changes from day to day. These so-called 'intrinsic fluctuations' [4] even happen spontaneously, i.e., in the absence of synaptic activity. Their exact function is still un- known. In addition to these spontaneous volume fluctuations, strong synaptic activity is able to specifically and permanently increase the volume of individual spine heads. Using glutamate uncaging (• Fig. 1), it was shown that these changes in the spine head volume represent the anatomical counterpart of synaptic potentiation [5, 6]. Structural and functional plasticity are therefore intertwined: the PSD of potentiated spines becomes enlarged as more AMPA receptors are inserted. Interestingly, the original size of a spine head is a good indicator of the degree of synaptic plasticity that can be induced. Synaptic potentiation of a large spine is not very effective and the spine head volume becomes only transiently enlarged. In contrast, small spines have the capacity to become permanently enlarged and potentiated. The fact that large spines are conserved over long timescales (sometimes for the entire lifetime of an animal) suggests that spines could be the elementary building blocks of memory: small spines are abundant, but not very stable. They tend to disappear with time unless they get potentiated. Since they are produced in large numbers, the nerve cell is able to form and to test many connections. If these synaptic contacts prove to be important, as indicated, e.g., by frequent temporal coincidence of presynaptic activity with postsynaptic depolarization, they will become potentiated and the spine head will grow. Spine structure can thus be viewed as a memory of recent coincidence events. This has interesting consequences: the history of activity of a synapse is represented in the size of the spine-old spines are big and carry strong synapses. This suggests that spines are not only binary storage elements, but also influence the lifetime of a memory trace: it is reasonable to assume that large spines are better protected against synapse loss and are therefore able to stabilize neuronal circuits they participate in.

The structural mechanism underlying spine motility as well as structural stabilization is polymerization and cross-linking of actin. Actin is the most abundant protein in spines and the sole cytoskeletal component present in all spines. Indeed, spine motility was first observed in live cells using fluorescently labeled actin
e-Neuroforum 2011 · 2:6-12

DOI 10.1007/s13295-011-0014-5

C) Springer-Verlag 2011

\section{J.S. Wiegert ·T.G. Oertner}

Shapeshifting for memory. Biochemical and electrical signaling in dendritic spines

\section{Abstract}

One of the biggest remaining mysteries of science is inside our heads: how does nature wire up a high-performance computer without having a detailed blueprint specifying the location and strength of every connection? It is assumed that local connectivity in our cortex is at first random, and during development undergoes refinement until only the 'right' connections are left over. But how can the brain tell 'right' from 'wrong' connections? The majority of excitatory connections are formed on dendritic spines, tiny excrescences that cover almost the entire dendritic surface of most neurons. Since their discovery by Ramón y Cajal in 1896, neuroscientists have been fascinated by these structures, which ultimately determine which neurons in the brain become connected and form functional networks. Here we review the many important functions of spines and explain why electrical and biochemical processes in these tiny structures are thought to be crucial for the plasticity of the brain.

Keywords Synaptic transmission · Learning · Long-term plasticity - Coincidence detection . Compartmentalization 


\section{Review article}
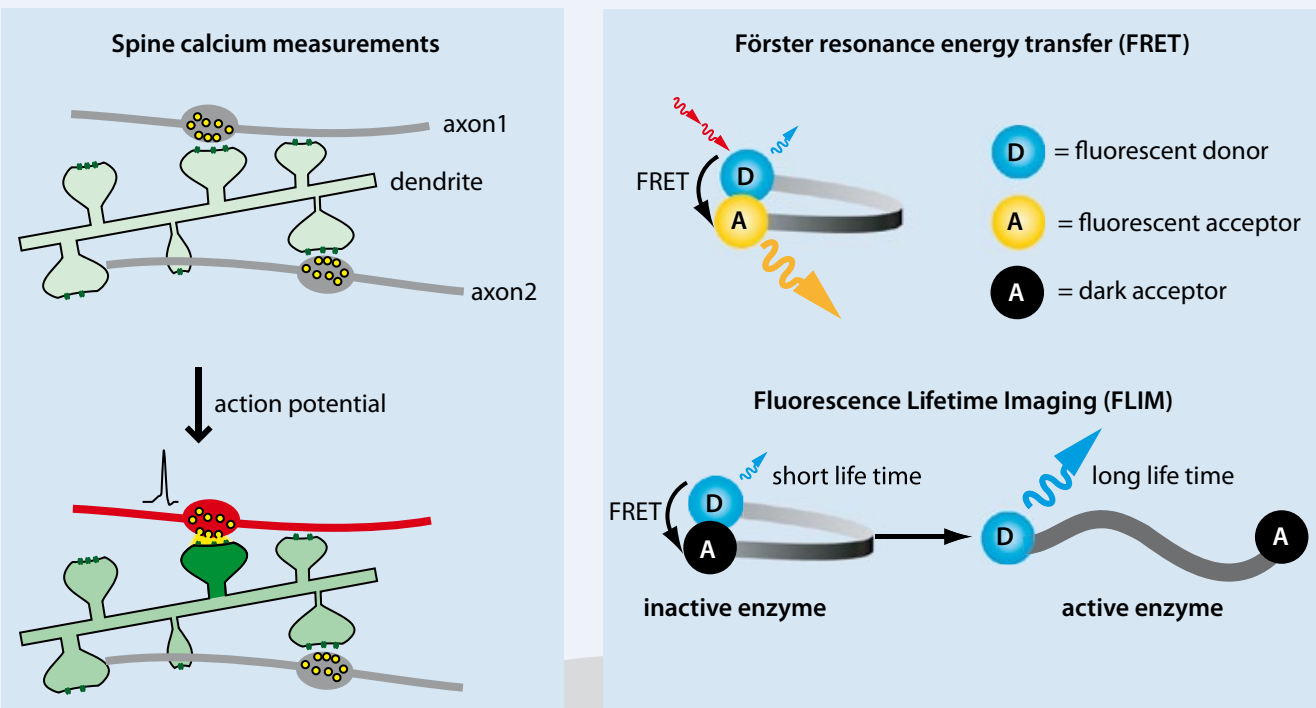

Principle of two-photon microscopy

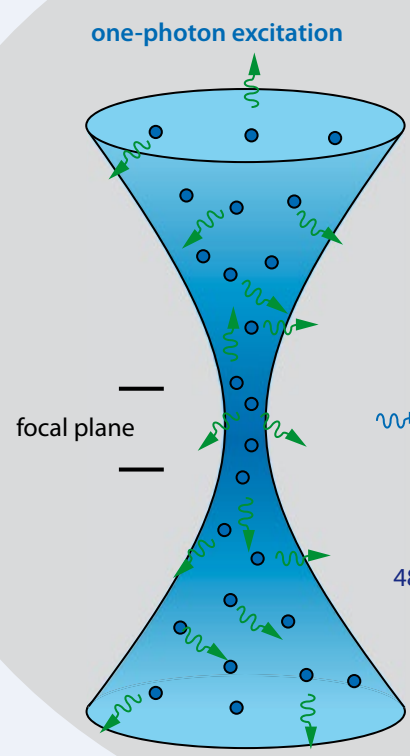

two-photon excitation

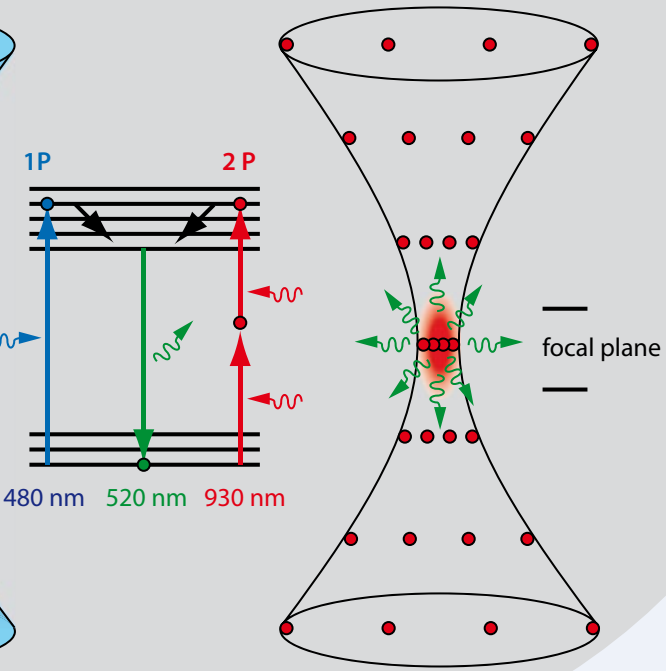

Local photolysis of caged glutamate

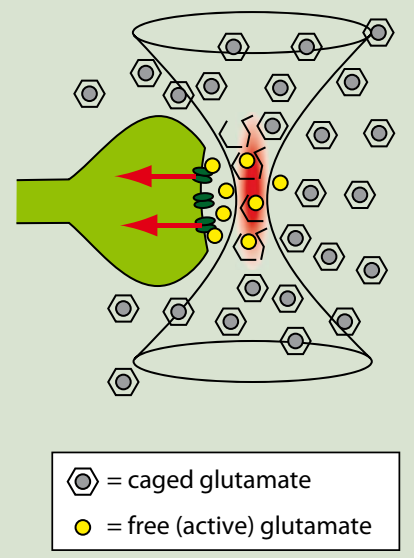

Fluorescence recovery after photobleaching (FRAP)

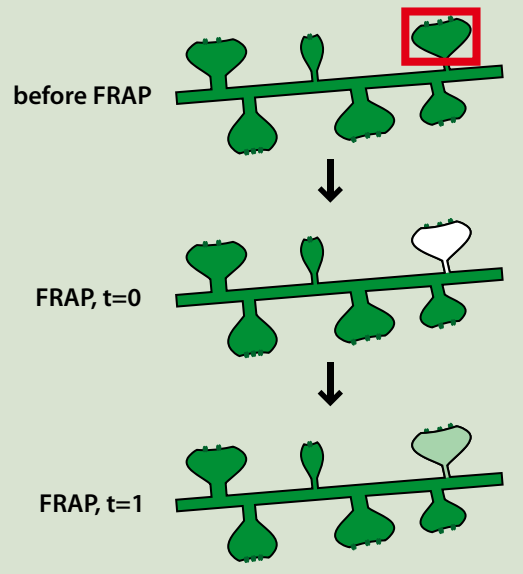


Fig. $1<$ Two-photon microscopy as a tool to study spine synapses. This technique can not only be used to image dendrites and spines, but also to probe the function of synapses. It is based on non-linear excitation of fluorophores by simultaneous absorption of two low-energy photons (700-1000 nm). Light of this wavelength (near-infrared) penetrates living tissue well and is not absorbed by endogenous proteins. Since the probability of two-photon excitation increases with the square of photon density, fluorescence is only generated in the very small volume of the laser focus (center, right). In order to reach sufficiently high photon densities, pulsed near-infrared lasers are used. Conventional (single-photon) excitation, in contrast, leads to fluorescence excitation throughout the cone of light (center, left). The energy diagram of a fluorescent molecule (center) depicts the difference between single- and two-photon excitation, resulting in an identical emission wavelength (green). By scanning the laser focus through the tissue, the fluorescence of a single plane can be measured point-by-point (optical sectioning). With this technique, it is possible to determine the calcium concentration inside a neuron (top, left). Using Förster resonance energy transfer (FRET) sensors (top, right), enzyme activity can be measured optically. FRET sensors are based on radiation-less energy transfer between a donor and an acceptor fluorophore. If the two fluorophores are attached to the ends of an enzyme molecule, FRET efficiency strongly decreases if the distance of the two fluorophores becomes more than a few nanometers. An elegant improvement of FRET is fluorescence lifetime imaging microscopy (FLIM). For this technique, the delay of donor fluorescence emission after a laser pulse is measured. FRET leads to a decrease in fluorescence lifetime and can thus be measured very accurately by FLIM. Since only donor fluorescence is evaluated, it can be advantageous to use a 'dark' (low quantum yield) acceptor. Further applications of twophoton excitation include light-induced release of biological signaling molecules (photolysis or 'uncaging') from a biologically inactive complex ('caged compound'). Due to the high spatial resolution and deep tissue penetration of two-photon excitation, signaling molecules can be released precisely and rapidly. Glutamate uncaging at single spines, for example, permits activation of single synapses without influencing neighboring spines. At the same time, calcium signals can be measured inside the spine. Measuring fluorescence recovery after photobleaching (FRAP, bottom right) reveals the dynamics and bound fraction of labeled molecules. FRAP and related methods (e.g., photoactivation) have contributed to our understanding of diffusional coupling between spines and dendrites
[3]. Filamentous actin polymers (f-actin) are unstable and undergo 'treadmilling, a constant depolymerization at one end and elongation at the other end of every actin filament. Almost all enzymes involved in the polymerization and depolymerization of actin are directly or indirectly calciumdependent. This explains why strong activation causes morphological expansion of the spine: when pre- and postsynaptic cells are active at the same time, a massive calcium influx in the spine triggers a wave of actin polymerization. Whether and how this transient expansion is transformed into a permanent increase in size is intensely debated and currently a very active area of research. One important player in the stabilization process seems to be calcium/calmodulin-dependent kinase II (CaMKII $\alpha$ ), an enzyme that also doubles as a structural component of the PSD [6].

Another interesting aspect of spines is the fact that they continuously exchange their molecular inventory during their lifetime. Even the most long-lived proteins of the PSD are replaced after about $3 \mathrm{~h}$ [7]. Judged by their recovery time constant after photobleaching (• Fig. 1), many other proteins have residence times of only a few minutes in the spine. Due to this high turnover, insertion of new glutamate receptors and other PSD proteins cannot be the whole explanation for the long-term stability of a spine. Since the functional status of a spine is maintained much longer than each individual molecular component, it is possible that activity-dependent processes contribute to the structural stability: if a spine is larger, it houses more glutamate receptors, leading to larger excitatory postsynaptic potentials (EPSPs). Strong depolarization produces large calcium transients within the spine, stimulating actin polymerization. Thus, an activity-dependent feedback loop could be responsible for spine stabilization. Whether morphological changes of spines are indeed the driving force for synaptic plasticity, or rather a by-product remains to be seen. What we have learned from recent experiments is that spines are not static biochemical units, but possess an active shape-changing mechanism to drive local structural changes in a highly regulated fashion.

\section{Spines as miniaturized biochemical reactors}

One of the most important functions of dendritic spines is the biochemical isolation of each synapse from its neighbors. Through this isolation, biochemical signaling cascades can be activated in individual synapses without disturbing chemical information processing in neighboring synapses. Following the development of two-photon microscopy by Winfried Denk and David Tank in 1990, it became possible to study spines in intact brain tissue. Rapid changes in intracellular calcium concentration (or fluorescently labeled proteins) can be observed optically in individual spines (• Fig. 1). When additional information about the volume of the spine is available, e.g., by fluorescently labeling the cytoplasm, the concentration of an enzyme can be mapped in a live neuron (• Fig. 2). Even when the neuron is not active, specific proteins (such as CaMKIIa or PSD95) are strongly enriched in spines.

In addition to the total concentration of a specific protein, it is often important to determine the fraction of molecules that are enzymatically active. Enzymes change their conformation when activated, and this conformational change can be exploited to bring two fluorophores into close contact. The result is a so-called FRET sensor of enzymatic activity, which can be genetically encoded and expressed in live neurons (• Fig. 1).

With these sensors, activation of signaling molecules could be monitored after activation of synapses on individual spines [8]. The result of these studies was intriguing: large, slowly diffusing enzymes like CaMKIIa inactivate long before reaching a neighboring spine. Small, long-lived signaling molecules like Ras, in contrast, are able to diffuse fast and thus transmit biochemical information between neighboring synapses. Apparently, biochemical isolation of spine synapses is not absolute, but has to be experimentally determined for each reaction. Adding to this complexity, computer simulations suggest that the morphology of individual spines further modifies the degree of compartmentalization and functional cross-talk between neighboring synapses. 

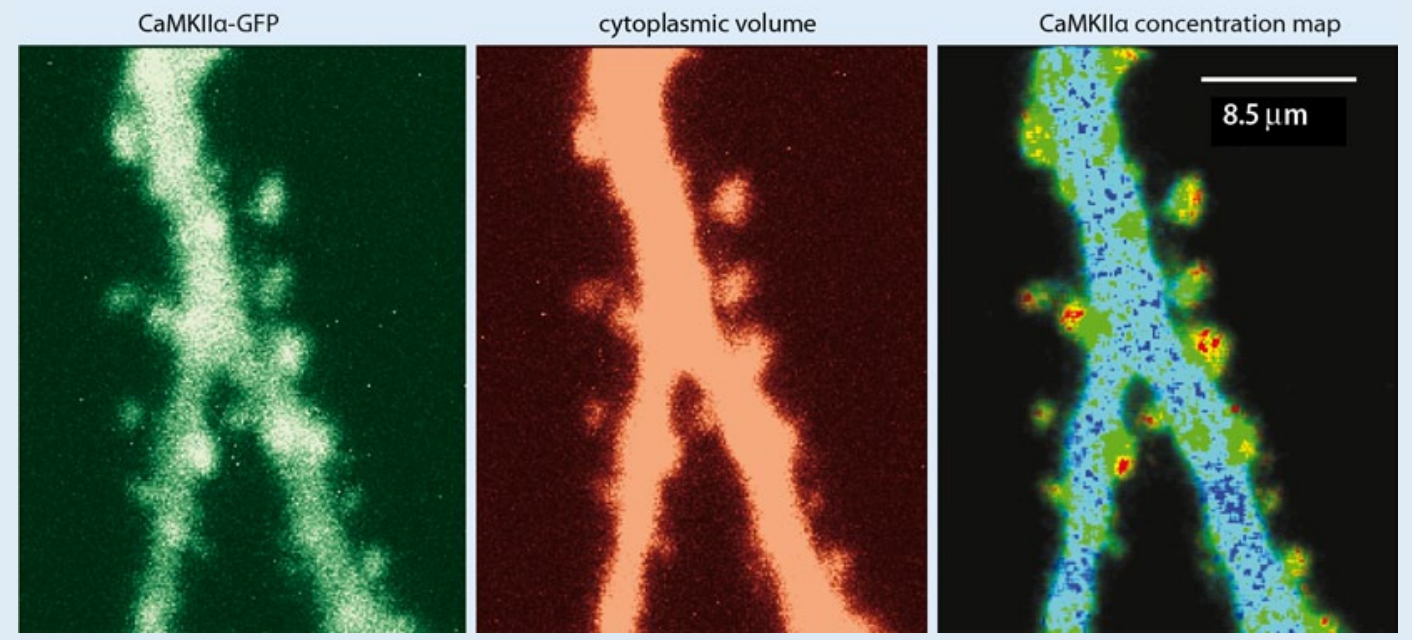

Fig. $2 \Delta$ Optical measurement of protein concentration in spines. Single neurons of an organotypic slice culture of the hippocampus were transfected with two proteins: a kinase coupled to a green fluorescent protein (CaMKIla-GFP, left) and a red fluorescent protein residing in the cytoplasm (RFP, center). The enzyme concentration is measured by the ratio of green to red fluorescence. In dendritic spines, this concentration is increased by $40 \%$ compared to the dendrite [6]. This suggests that many kinase molecules are already postsynaptically anchored in their inactive state

In summary, spines are able to sense temporally correlated electrical activity via changes in local calcium concentration. Inside the spine, this information is transformed into longer-lived biochemical signals (e.g., CaMKIIa activation, actin polymerization, AMPA receptor insertion). Thus, biochemical compartmentalization through spines leads to a restriction of synaptic plasticity to the places where the cell received correlated input. This specificity is crucial, since every neuron receives inputs from tens of thousands of other neurons. If large networks of neurons indeed act as information storage matrices, it is desirable to modulate the strength of specific nodes while leaving other connections untouched.

\section{Spines as electrical compartments}

As explained in the previous section, biochemical isolation of individual synapses has been demonstrated in a large number of experiments and is considered one of the most important functions of spines. In the 1980s, it was suggested that spines could also have electrical functions [9]. Several theoretical studies were inspired by the discovery that the dendrites of neurons are not electrically passive devices, but rather have a multitude of voltage-gated channels and therefore actively modulate the amplitude of postsynaptic potentials. Due to their small size, spines have an extremely low capacitance and a high input resistance. This allows for fast and strong depolarization by synaptic currents. In addition, spine depolarization can be further amplified by voltage-gated calcium or sodium channels in the spine [10]. NMDA receptors, due to their pronounced voltage-dependence, also contribute to this amplification. Whether or not a specific spine is sufficiently isolated from its dendrite to constitute an independent electrical compartment depends largely on the electrical resistance of the spine neck. The question of spine neck resistance, however, is highly controversial. Measurements of the spine neck diameter and its diffusional resistance have led to the conclusion that the electrical resistance of the spine neck is negligible [11, 12]. In contrast, optical measurements of membrane potential indicate that spines have different electrical properties than the attached dendrites and therefore have at least some degree of autonomy [13], pointing to relatively high spine neck resistances. In glutamate uncaging experiments (• Fig. 1), the length of the spine neck was inversely correlated with the amplitude of the electrical signal at the soma [14], indicating that at least in some spines, synaptic potentials are strongly filtered by the spine neck.
What could be the reason for this disagreement on the 'typical' spine neck resistance? First, absolute measurements of spine head depolarization using voltagesensitive dyes are very difficult to calibrate. Second, spine necks are surprisingly plastic and can change their diffusional resistance within minutes [15]. As a consequence, slightly different experimental procedures or preparations can dramatically alter resistance estimates. Third, correlative datasets from light and electron microscopy (• Fig. $\mathbf{3}$ ) suggest that the cytoplasm in the spine has very different physical properties compared to dendritic cytoplasm. Estimates of electrical resistance based on spine morphology, however, assume the entire neuron is filled with homogenous cytoplasm. Until more information about the plasticity and the internal structure of the spine neck becomes available, it is safe to say that there is certainly a large variability of spine neck resistance in individual neurons, and, in addition, systematic differences between preparations (acute brain slice vs. in vivo) exist.

\section{Spines detect causality and influence the plasticity of synapses}

After this discussion of biophysical properties of spines, we would like to return 

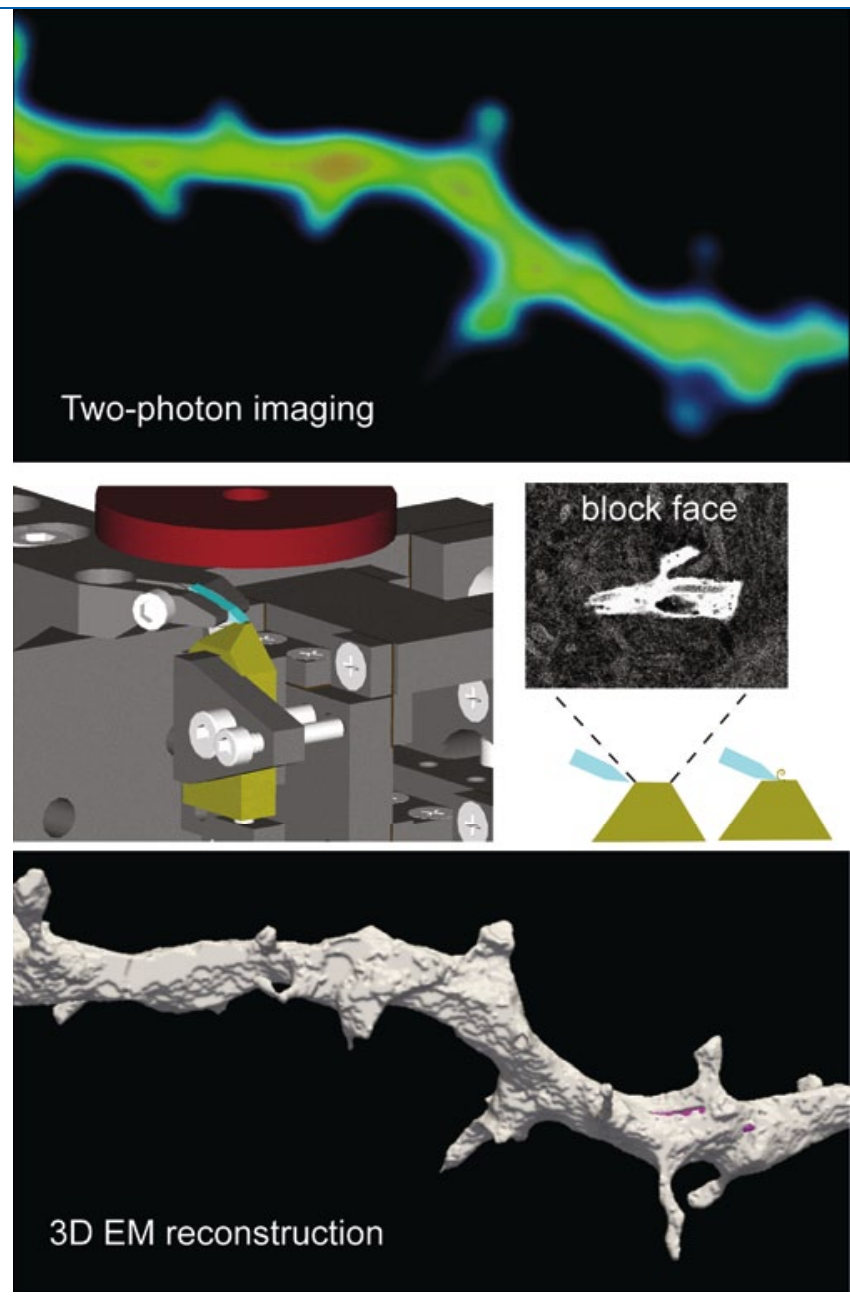

Fig. $3 \Delta$ Correlative microscopy of dendritic spines. We observed spines in a living cell with two-photon microscopy [21] and measured the diffusional resistance by targeted bleaching of fluorescence. The tissue was fixed afterwards, embedded in a synthetic resin, and automatically cut in a scanning electron microscope. This technique is known as serial block-face scanning [22]. In the scheme, the tissue block (yellow) and the moving diamond knife (blue) is drawn. The red disk depicts the detector of back-scattered electrons (modified from [22]). With the resulting three-dimensional reconstruction, the morphology of single spines can be measured and correlated with previously measured properties (C. Vivien, C. Genoud). As synapses are at the resolution limit of light microscopy, the combination with electron microscopy is a major advantage

to our initial question: how does the brain distinguish between 'wrong' and 'right' synapses? The Canadian psychologist Donald Hebb proposed in 1949 that synaptic connections should be strengthened if pre- and postsynaptic cells were active in rapid succession. This rule would favor all synapses that participated in the initiation of postsynaptic action potentials. This proposition was later experimentally confirmed and is now known as Hebb's rule. What is the precise mechanism of synaptic coincidence detection? It has been shown recently that three sig-

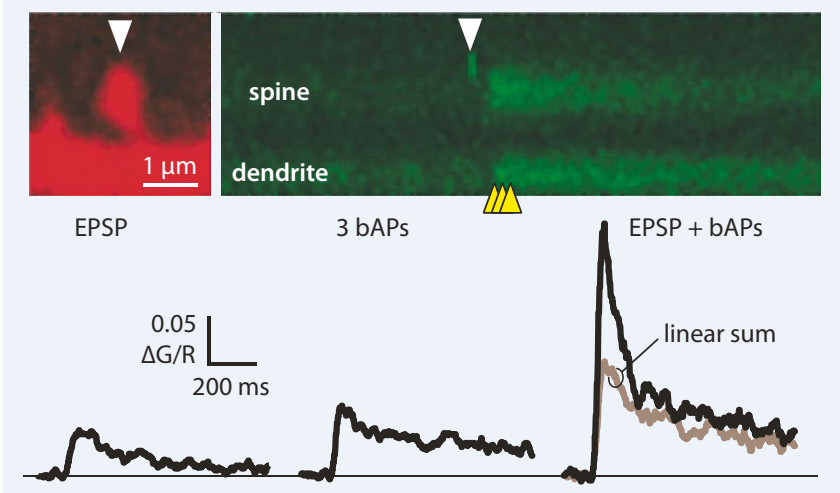

Fig. $4 \Delta$ Calcium measurements during coincident activity in spines. Three action potentials (yellow arrows) were triggered in the dendrite after targeted glutamate uncaging at synaptic cleft (arrow). The resulting calcium signal in the spine (black curve, right) is much larger than predicted by linear summation of the single components (gray curve). This type of stimulation not only leads to huge calcium signals in the spine but also to long-term potentiation of this synapse [16]

PA receptors gate this calcium influx and provide temporal precision [16]. Highly supralinear calcium influx is observed only in diffusionally well isolated spines, suggesting that spines might have evolved specifically to allow coincidence detection with high temporal precision (in the order of milliseconds). As discussed above, calcium-dependent enzymes are retained in the spine, close to the activated synapse $[6,17]$. As the geometry of spines is highly variable and influences diffusion processes as well as input resistance, it is likely that every synapse has its own threshold for potentiation.

For all its explanatory power, Hebb's rule poses logical problems since it provides for a positive feedback mechanism only: strong synapses are more likely to participate in the generation of postsynaptic action potentials and, as a result, would be increasingly strengthened. Of course nature has devised ways to reduce synaptic strength and to prevent such runaway potentiation. One such mechanism is synaptic depression after activation of metabotropic glutamate receptors (mGluR-LTD). Interestingly, this 'synaptic emergency brake' is only present in spines containing strong synapses, counteracting the Hebbian mechanism at these synapses. We have shown that induction of mGluR-LTD depends on the presence of endoplasmic reticulum (ER) in spines, which releases large amounts of calcium after repeated activation of mGluR receptors [18]. Since the ER is not present in all 
spines, neighboring synapses on the same dendrite display different forms of plasticity.

Finally, we would like to emphasize that the 'typical spine' is merely a theoretical construct. What makes spines so interesting is precisely their variability. While it is well established that the size of a spine is a good proxy for the strength of its synapse, we are just beginning to understand how the shape of a spine and organelles inside its head enable various forms of long-term plasticity. In this context, it is not surprising that human diseases leading to mental retardation are often accompanied by aberrant spine morphology [19]. Studying biochemical signaling cascades in single spines 'in situ' remains technically challenging, but advances in microscopy and the development of genetically encoded indicators of protein function make complex experiments possible. Recent experiments suggest that through observation of spines it might become possible to track down memory traces in the brain, one of the holy grails of neurobiology [20]. We need to unravel the regulation of individual synapses to understand how the wiring of the brain becomes optimized during development and how we are able to store memories over years.

\section{Corresponding address \\ J.S. Wiegert}

Friedrich Miescher Institute for Biomedical

Research

Maulbeerstr. 66, 4058 Basel

Switzerland

simon.wiegert@fmi.ch

J.S. Wiegert 2005: received his diploma in biology from the Ruprecht-Karls University, Heidelberg. 2005-2009: $\mathrm{PhD}$ thesis in the group of Prof. Dr. Hilmar Bading (Interdisciplinary Center for Neurosciences, University of Heidelberg). At present, Simon Wiegert is a postdoctoral fellow at the Friedrich Miescher Institute for Biomedical Research (FMI) in Basel. In 2010, he received a Marie Curie Fellowship (Seventh Framework Programme, EU).

\section{T.G. Oertner}

Friedrich Miescher Institute for Biomedical Research

Maulbeerstr. 66, 4058 Basel

Switzerland

thomas.oertner@fmi.ch
T.G. Oertner 1997: received his diploma in biology from the Albert-Ludwigs University, Freiburg. 1997-2000: $\mathrm{PhD}$ thesis in the group of Prof. Dr. Alexander Borst (Friedrich-Miescher Laboratory of the Max-Planck Society, Tübingen). 2000-2003: Postdoctoral training in the group of Prof. Dr. Karel Svoboda (Cold Spring Harbor Laboratory, NY, USA). 2003-2009: Junior group leader at the Friedrich Miescher Institute for Biomedical Research (FMI) in Basel, Switzerland. In 2009, Thomas Oertner was appointed Senior Scientist at the FMI in Basel. Fellowships: 1997-1999 Member of the Graduiertenkolleg Neurobiology (University of Tübingen); 2000-2001 postdoctoral fellowship from the Swartz Initiative for Computational Neuroscience (Stony Brook, NY, USA). 2005-2008 on the faculty of the summer course Neurobiology (MBL, Woods Hole, MA, USA).

\section{References}

1. Matsuzaki M, Ellis-Davies GC, Nemoto T et al (2001) Dendritic spine geometry is critical for AMPA receptor expression in hippocampal CA1 pyramidal neurons. Nat Neurosci 4:1086-1092

2. Holtmaat AJ, Trachtenberg JT, Wilbrecht L et al (2005) Transient and persistent dendritic spines in the neocortex in vivo. Neuron 45:279-291

3. Fischer M, Kaech S, Knutti D et al (1998) Rapid actin-based plasticity in dendritic spines. Neuron 20:847-854

4. Yasumatsu N, Matsuzaki M, Miyazaki T et al (2008) Principles of long-term dynamics of dendritic spines. J Neurosci 28:13592-13608

5. Matsuzaki M, Honkura N, Ellis-Davies GC et al (2004) Structural basis of long-term potentiation in single dendritic spines. Nature 429:761-766

6. Zhang YP, Holbro N, Oertner TG (2008) Optical induction of plasticity at single synapses reveals input-specific accumulation of alphaCaMKII. Proc Natl Acad Sci U S A 105:12039-12044

7. Gray NW, Weimer RM, Bureau I et al (2006) Rapid redistribution of synaptic PSD-95 in the neocortex in vivo. PLoS Biol 4:e370

8. Lee SJ, Escobedo-Lozoya Y, Szatmari EM et al (2009) Activation of CaMKII in single dendritic spines during long-term potentiation. Nature 458:299-304

9. Segev I, Rall W (1988) Computational study of an excitable dendritic spine. J Neurophysiol 60:499523

10. Bloodgood BL, Sabatini BL (2007) Ca(2+) signaling in dendritic spines. Curr Opin Neurobiol

11. Harris KM, Stevens JK (1989) Dendritic spines of CA 1 pyramidal cells in the rat hippocampus: serial electron microscopy with reference to their biophysical characteristics. J Neurosci 9:2982-2997

12. Svoboda K, Tank DW, Denk W (1996) Direct measurement of coupling between dendritic spines and shafts. Science 272:716-719

13. Palmer LM, Stuart GJ (2009) Membrane potential changes in dendritic spines during action potentials and synaptic input. J Neurosci 29:6897-6903

14. Araya R, Jiang J, Eisenthal KB et al (2006) The spine neck filters membrane potentials. Proc Natl Acad Sci U S A 103:17961-17966

15. Grunditz A, Holbro N, Tian L et al (2008) Spine neck plasticity controls postsynaptic calcium signals through electrical compartmentalization. J Neurosci 28:13457-13466

16. Holbro N, Grunditz A, Wiegert JS et al (2010) AMPA receptors gate spine $\mathrm{Ca}(2+)$ transients and spiketiming-dependent potentiation. Proc Natl Acad Sci U S A 107:15975-15980
17. Oertner TG (2009) How do synapses measure milliseconds? Front Comput Neurosci 3:7

18. Holbro N, Grunditz A, Oertner TG (2009) Differential distribution of endoplasmic reticulum controls metabotropic signaling and plasticity at hippocampal synapses. Proc Natl Acad Sci U S A 106:15055-15060

19. Irwin SA, Galvez R, Greenough WT (2000) Dendritic spine structural anomalies in fragile- $X$ mental retardation syndrome. Cereb Cortex 10:1038-1044

20. Xu T, Yu X, Perlik AJ et al (2009) Rapid formation and selective stabilization of synapses for enduring motor memories. Nature 462:915-919

21. Oertner TG (2002) Functional imaging of single synapses in brain slices. Exp Physiol 87:733-736

22. DenkW, Horstmann H (2004) Serial block-face scanning electron microscopy to reconstruct three-dimensional tissue nanostructure. PLoS Biol 2:e329 